\title{
PAPR Reduction Technique for High Speed Application
}

\author{
Kendre Anuradha T. \\ Department of Electronics Engineering \\ M.S.Bidve Engineering College \\ Latur-413531 Maharashtra
}

\author{
Suresh R Halhalli \\ Head, Department of EE \\ M.S.Bidve Engineering College \\ Latur-413531 Maharashtra
}

\begin{abstract}
Orthogonal frequency division multiplexing (OFDM) is a robust and effective multicarrier transmission technique for high speed communication in wireless mobile environment and applications. A major challenging issue in application of OFDM is its high peak to average power ratio (PAPR). Partial transmit sequence (PTS) scheme is an attractive technique for PAPR reduction in OFDM systems. In conventional PTS, an exhaustive search for phase sequences is needed to obtain desirable PAPR performance, which results in high computational complexity. In this paper, a hybrid combination of tree PTS (T-PTS) algorithm and real part-imaginary part (RP-IP) separation technique is proposed. The simulation results of the proposed technique show improved PAPR performance with remarkably reduced bit error rate (BER), over all variants of PTS. Also, the technique offers significant reduction in computational complexity over conventional PTS technique.
\end{abstract}

\section{Keywords}

Orthogonal Frequency Division Multiplexing (OFDM), Peak to average power ratio (PAPR), Partial transmit sequence (PTS).

\section{INTRODUCTION}

OFDM is widely implemented in various high speed wireless communication standards because of its favorable properties such as high spectral efficiency, robustness to channel fading, immunity to impulse interference and capability of handling multipath fading.

OFDM is a multicarrier modulation technique, which employs several carriers, within the allocated bandwidth, to convey the information from source to destination. Each carrier may employ one of the several available digital modulation techniques (BPSK, QPSK or QAM) [1]. A sampled time domain sequence $x_{n}=\left[x_{0} x_{1} x_{2}, \cdots, x_{(N-1)}\right]^{T}$ in OFDM system is obtained by performing an $N$-point IFFT on frequencydomain sequence $\mathrm{X}_{\mathrm{k}}=\left[\mathrm{X}_{0}, \mathrm{X}_{1}, \cdots, \mathrm{X}_{(\mathrm{N}-1)}\right]^{\mathrm{T}}$.

Where, the time domain signal is defined as:

$$
\mathrm{x}_{\mathrm{n}}=\frac{1}{\mathrm{~N}} \sum_{\mathrm{k}=0}^{\mathrm{N}-1} \mathrm{X}_{\mathrm{k}} \mathrm{W}_{\mathrm{N}}^{-\mathrm{nk}} \quad 0 \leq n \leq N-1
$$

Where, $\mathrm{x}_{\mathrm{n}}=$ Time domain signal.

$W_{N}^{n k}=\exp (-j 2 \pi n k / N)$,

$K$ is the frequency index and $\mathrm{n}$ is the time index.

Equation (1) can be thought of as an Inverse Fast Fourier Transform (IFFT) where ' $N$ ' is the size of IFFT. To acquire the original transmitted signal, FFT is performed at the receiver side [2].

In spite of the several advantages offered by OFDM, there is a limitation of high PAPR, which can be reduced using several techniques such as clipping and filtering [3], companding [4], coding [5], selective mapping (SLM) [6], partial transmit sequence (PTS) [7-9]. PTS technique improves PAPR statistics of an OFDM signal significantly without any in-band distortion and out-of-band radiation [10].

The conventional PTS offers reduction in PAPR at the cost of higher computational complexity. By using different PTS techniques such as Tree PTS (T-PTS) [11] and RP-IP PTS [12], better reduction in PAPR and BER can be achieved with considerable reduction in computational complexity.

In this work a hybrid combination of T-PTS algorithm and RP-IP PTS is proposed for PAPR reduction of OFDM signal. The proposed technique mainly concentrates on reducing the PAPR and BER significantly over that offered by the individual techniques. The computational complexity is a major issue for implementation of PTS, which is also reduced considerably compared to that of conventional PTS. Next section presents the introduction to PAPR. Section- 3 give the details of PTS techniques to reduce the PAPR. The details of proposed techniques are presented in section- 4 and section- 5 presents the simulation results. Finally conclusions are drawn in section-6.

\section{PEAK TO AVERAGE POWER RATIO}

OFDM has several features which make it an attractive modulation scheme for high speed transmission links. However, one major limitation is its high Peak to Average Power Ratio (PAPR). These large peaks cause saturation in power amplifiers at the transmitting end, leading to intermodulation among the subcarriers, which causes an increase in the out of band (OOB) energy. Hence, to design a cost effective and robust system, it is highly desirable to reduce the PAPR.

The mathematical representation of PAPR of an OFDM signal is given as -

$$
\operatorname{PAPR}\left[x_{n}\right]=\frac{0 \leq n<N\left|x_{n}\right|^{2}}{E\left[\left|x_{n}\right|^{2}\right]}
$$

Where E [.] denotes average power. PAPR can be expressed in ' $\mathrm{dB}$ ' as follows,

$$
\operatorname{PAPR}(d B)=10 \log _{10} \operatorname{PAPR}\left(x_{n}\right)
$$


Among the different techniques proposed in literature, PTS technique require low computational complexity in which selection of proper phase sequences is done to achieve significant PAPR reduction [3].

\section{PARTIAL TRANSMIT SEQUENCE}

PTS method is a distortion less probabilistic technique for PAPR reduction. This technique is called distortion less because the quantity with which the actual signal altered is sent as side information.

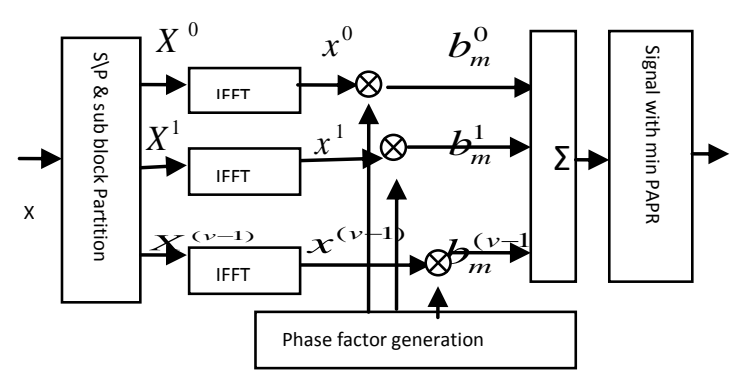

Fig. 1 Block Diagram of Basic PTS [14]

In conventional PTS [14], the frequency-domain sequence $X$ is partitioned into $V$ disjoint sub-blocks as shown in Fig.1.

$$
X^{(v)}=\left[X_{0}^{(v)}, X_{1}^{(v)}, \cdots, X_{N-1}^{(v)}\right]^{T}
$$

Where, $v=0,1, \cdots, V-1$

In every sub-block, only $\mathrm{N} / \mathrm{V}$ signal points exist and the others are set to zero [7]. Then, $V$ sub-blocks $X^{(v)}$ are combined with phase factor $b_{m}=\left[b_{m}^{(0)}, b_{m}^{(1)}, \cdots, b_{m}^{(v-1)}\right]^{T}$ vector According to [3].

$$
\begin{aligned}
& X_{m}=\left[X_{0}^{m}, X_{1}^{m}, \cdots, X_{N-1}^{m}\right]^{T} \\
& =\left[X_{0}^{(0)}, X_{1}^{(1)}, \cdots, X_{(N-1)}^{(V-1)} b_{m}\right.
\end{aligned}
$$

Where the phase factor $b_{m}^{(v)}$ belongs to phase factor set $\Phi=\left\{e^{j \phi_{1}}, \cdots, e^{j \phi_{w}}, \cdots, e^{j \phi_{w}}\right\}, \phi_{w} \in[0,2 \pi], \quad W \quad$ is the number of elements in the phase factor set, $\mathrm{m}=1,2 \ldots \mathrm{M}$. M is the number of used phase factor vectors. Because the first element in every phase factor vector $b_{m}^{(0)}=1$ can be fixed without loss of PAPR performance, the number of phase factor vectors is $M=W^{(v-1)}$ generally, in order to reduce the computational complexity, the phase factor set is restricted to $\{1,-1, j,-j\}$.Subsequently, an N-point IFFT is performed on $X_{m}$ to generate a time-domain candidate $x_{m}$

$$
x_{m}=\operatorname{IDFT}\left(X_{m}\right)
$$

If $\mathrm{M}$ phase factor vectors are used, $\mathrm{M}$ time-domain candidates can be obtained. For one OFDM symbol, the number of needed IFFTs is equal to the number of candidates [13-15].

\subsection{RP-IP Separation in PTS}

As shown in fig. 2 the real part and imaginary part of the IFFT signal is multiplied with the corresponding real and imaginary part of the phase vector respectively $[7,12]$. This results in increased number of candidate signals. However, the computational complexity is reduced in this technique.

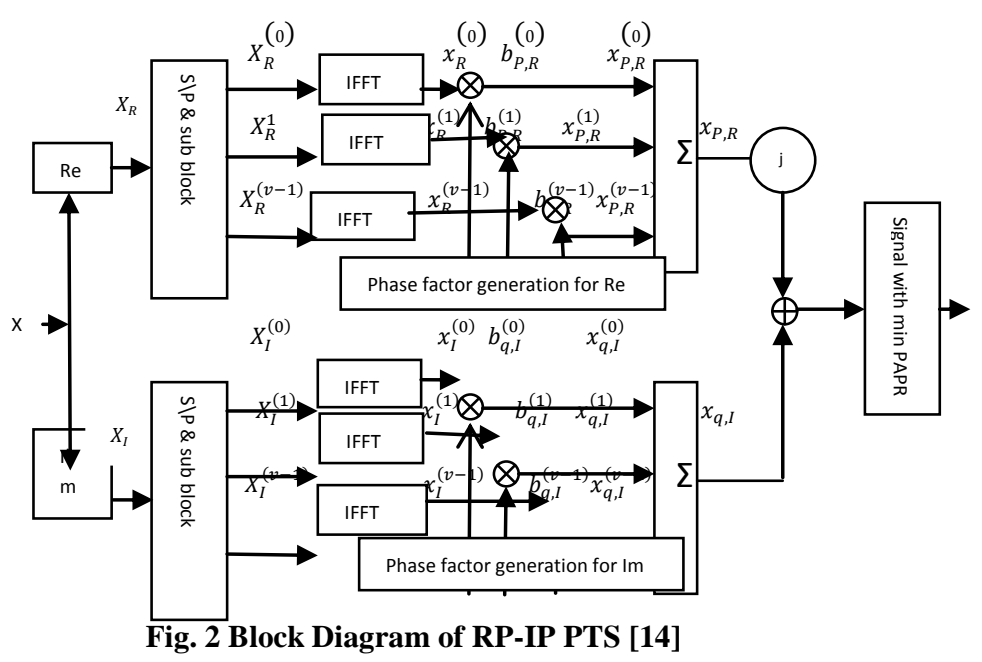

In this method, the number of required IFFTs is $2 \mathrm{M}$. If $\mathrm{M}$ is larger, the computational complexity of IFFTs is considerably high. For one OFDM symbol, 2V sets of N-points IFFTs are needed, which include, $V$ sets of IFFTs for real part operation, and $\mathrm{V}$ sets of IFFTs for imaginary part operation. In conventional PTS, IFFT is performed on the modulated signal which is a complex quantity whereas here a real valued signal is processed; hence number of multiplications needed for IFFT are reduced. The complexity of $2 \mathrm{~V}$ real-to-complex IFFTs is nearly the same as that of V complex IFFTs, as parallel operations are performed.

\subsection{Tree PTS Technique (T-PTS)}

Conventional PTS becomes impractical for implementation in high speed data transmission systems because of its very high computational complexity. An exhaustive search for the appropriate phase sequence has to be performed. The Tree PTS technique reduces the number of computations required to obtain the optimum phase sequence for an OFDM symbol.

Following steps are followed for Tree PTS [11]:

1. The modulated symbols are partitioned into $\mathbf{M}$ disjoint sub blocks.

2. Initially the phase vector is set to $\{11 \ldots 1\}$.

3. As the phase values are restricted to $\{1,-1, \mathrm{j},-\mathrm{j}\}$, we alter the phase vector for the first sub block to these values.

4. PAPR values are calculated for each combination and the minimum PAPR value is found.

5. Select the phase value which gives the minimum PAPR.

6. Fix this Phase value for first sub block.

7. Repeat the steps 4 to 6 till the $\mathrm{M}^{\mathrm{Th}}$ sub block.

The following example illustrates the execution of the above algorithm:

Consider first sub block out of M sub blocks. Calculate PAPR of the OFDM signals after rotating phase of the first sub block using $\mathrm{W}$ phase factors. $\mathrm{PAPR}_{1}$ is the PAPR value obtained after multiplication of first sub block with first phase vector. Similarly other PAPR values are obtained.

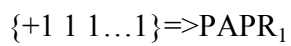

$$
\begin{aligned}
& \left\{\begin{array}{llll}
-1 & 1 & 1 & \ldots
\end{array}\right\}=>\text { PAPR }_{2} \\
& \{+\mathrm{j} 11 \ldots 1\}=>\text { PAPR }_{3}
\end{aligned}
$$




$$
\{-\mathrm{j} 11 \ldots 1\}=>\text { PAPR }_{4}
$$

Minimum PAPR is obtained from the above four values.

Min. \{PAPR1, PAPR2, PAPR3, PAPR4\}. Let PAPR3 be the minimum value obtained and hence fix ' $+\mathrm{j}$ ' as phase value for first sub block.

Similarly, other phase values are obtained for other sub blocks. Final phase sequence is traced according to tree algorithm [11, 16].

Example shown in Fig. 3 may help to understand the details of the T-PTS Scheme. Initially, consider the PAPR1 (8.3467dB) with all phase factors as 1's, now by changing the first value of phase factor as $\{1,-1, \mathrm{j},-\mathrm{j}\}$ four PAPR values are obtained, out of which the minimum value is selected. The original PAPR of OFDM signal in this example is $8.3467 \mathrm{db}$. This PAPR is reduced to $7.0476 \mathrm{~dB}$ by selecting $\mathrm{j}$ in the first sub block, and reduced to $6.8635 \mathrm{~dB}$ by selecting $\mathrm{j}$ in the second sub block. The value of PAPR is maintained in the third sub block obviously by selecting 1 and finally it is reduced to $6.4869 \mathrm{~dB}$ by selecting $\mathrm{j}$ in the fourth sub block. Thus the final decision of phase factors for minimum PAPR is $\{-\mathrm{j}, \mathrm{j}, 1, \mathrm{j}\}$

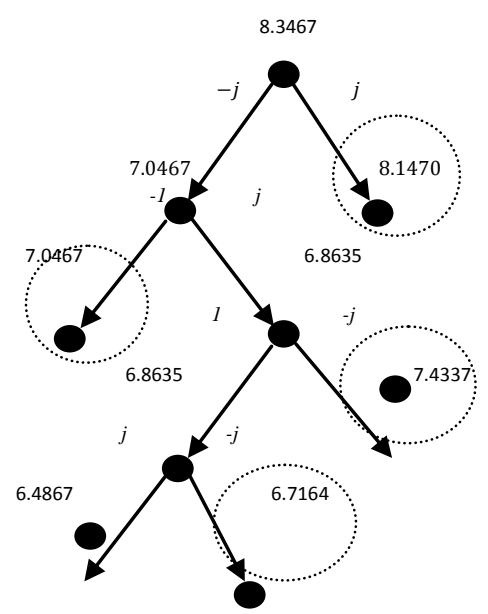

Fig. 3 Tree structure of the T-PTS scheme

\section{PROPOSED TECHNIQUE}

High computational complexity, due to search of phase vectors through a high-dimensional vector space, is a potential problem for practical implementation of PTS. To address the complexity issue, a hybrid combination of T-PTS along with this work.

This technique adaptively generates the real and imaginary phase factors separately by using Tree searching algorithm. The phase vector is chosen such that the real part of the signal is multiplied with real part of the phase vector and similar operations are done for imaginary part of the IFFT signal. The possible combinations for real phase vector will be

$\{1,-1\}$.Similarly, for imaginary phase vector possible combinations are given as: $\{\mathrm{j},-\mathrm{j}\}$

Following steps are followed in this hybrid technique:

1. The modulated signals are partitioned into M sub blocks.

2. IFFT operation is performed on each sub block.

3. Separate real and imaginary parts of all sub blocks. Let them be called RP and IP.

4. Initially, set the phase vector as all ones $\{1,1 \ldots 1\}$.
5. While finding phase vector for a particular sub block, keep all phase vectors equal to ' 1 ' for RP and ' $\mathrm{j}$ ' for IP.

6. For the first sub block, change the phase vectors from ' 1 ' to '-1' for RP and ' $\mathrm{j}$ ' to ' $-\mathrm{j}$ ' for IP.

7. Four combinations of the phase vectors along with the first sub block are obtained as follows:

Combination no. 1: $\mathrm{RP} * 1+\mathrm{IP} * \mathrm{j}$

Combination no. 2: $\mathrm{RP} *-1+\mathrm{IP} * \mathrm{j}$

Combination no. 3: RP* $1+$ IP $*_{-j}$

Combination no. 4: RP* $*-1+\mathrm{IP}^{*}-\mathrm{j}$

8. Add the newly formed version of the first sub block with the other sub blocks which are kept unchanged and find PAPR of the combination.

9. Find minimum PAPR out of the above four combinations and select phase vector corresponding to that symbol accordingly.

For example, if Combination no. 3 gives least PAPR for a particular sub block, then the phase vector for that sub block becomes $\{1,-j\}$.

10. Thus, PAPR reduction is due to the change in the considered sub block only, as other sub blocks are kept unchanged.

11. Fix the obtained phase value for the first sub block and accordingly alter the phase vector.

12. Repeat the steps 3 to 8 up to the MTh sub block.

13. The final phase vector approaches the optimum PAPR value at the end of the MTh sub block.

\section{SIMULATION RESULT}

System specifications used for unmodified OFDM, conventional PTS, RP-IP separation PTS, T-PTS and proposed Hybrid PTS technique are specified in Table 1.The simulation is performed using MATLAB.

During transmission, the OFDM data block is sent first and the side information related to that particular data block follows on a separate set of frequencies. This keeps the PAPR of the data signal unaltered even after addition of side information to the transmitted signal.

\section{TABLE 1: Specifications}

\begin{tabular}{|c|c|c|}
\hline $\begin{array}{c}\text { Sr. } \\
\text { No. }\end{array}$ & Simulation Parameters & $\begin{array}{c}\text { Parameter } \\
\text { Values }\end{array}$ \\
\hline 1 & Number of input bits & $1,00,000$ \\
\hline 2 & Modulation for data symbols & 16 QAM \\
\hline 3 & Modulation for side information & 8 QAM \\
\hline 4 & No. of bits per symbol & 4 \\
\hline 5 & Oversampling Rate & 4 \\
\hline 6 & No. of sub-blocks (M) & 4 \\
\hline 7 & IFFT/FFT size (N) & 256 \\
\hline 8 & IFFT/FFT size for side information & 16 \\
\hline
\end{tabular}

The execution time taken for conventional PTS is the highest of all, as it searches for all possible combinations of the phase vectors thereby increasing the computational complexity. In this work, the proposed technique offers nearly 40 times reduction in computational complexity over conventional PTS.

The CCDF plot in Fig.4 shows PAPR reduction for each individual PTS technique. It can be interpreted from Fig. 4 
that the proposed technique shows PAPR reduction better than that of the basic PTS and T-PTS. A PAPR reduction of nearly $3 \mathrm{~dB}$ is obtained, as compared to that of basic OFDM.

The proposed technique shows excellent performance in reception of the data even after it is passed through the AWGN channel. As compared to the other techniques considered in this experimentation, maximum reduction in the BER is offered by the proposed technique ( shown in Fig. 5).

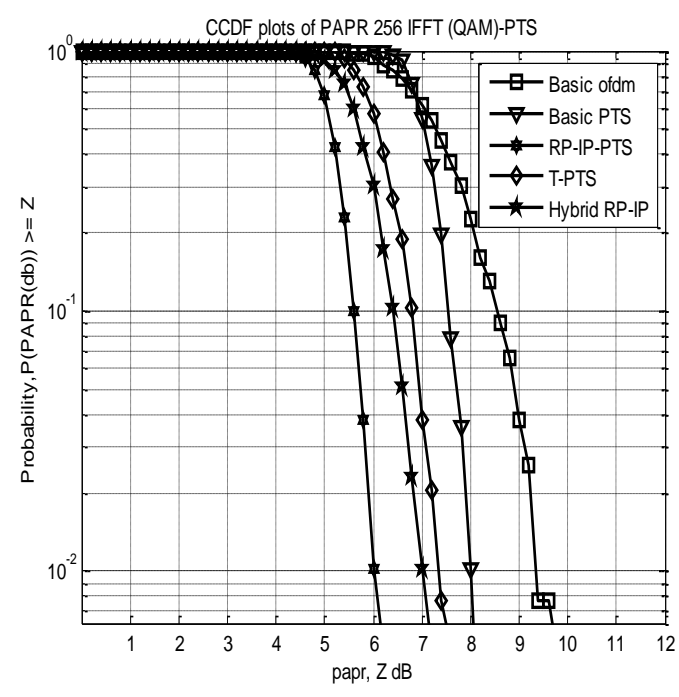

Fig. 4. CCDF plots for different PTS techniques

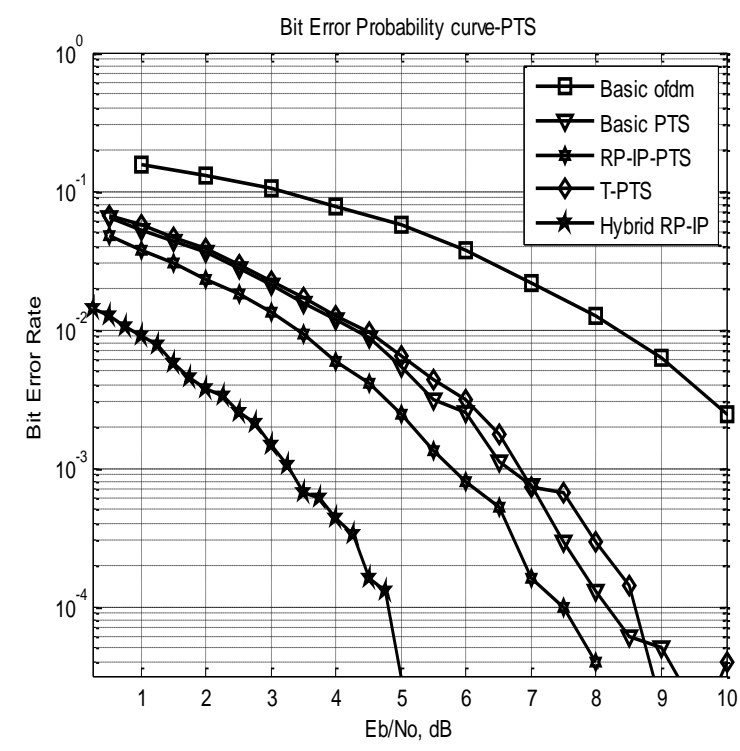

Fig. 5. BER plots for different PTS techniques

In an OFDM system, PAPR of the system is one of the major concern, but accurate reception of data is equally important. At the same time, the computational complexity of the PTS system has to be reduced so as to make the system practicaly realisable. All these aspects are precisely taken care of in the proposed technique in this paper.

\section{CONCLUSION}

In this paper, a modification in the conventional PTS scheme is proposed. The proposed technique of hybrid combination of Tree-PTS and RP-IP separation shows a significant reduction in PAPR as compared to that offered by individual techniques.
Computational complexity is reduced by a large factor than the conventional PTS. The proposed technique offers better BER performance than that of all variants of PTS techniques under consideration

\section{ACKNOWLEDGEMENT}

We convey our sincerer thanks to the principal faculty and staff of M.S.Bidve Engineering College Latur for help in carrying out this research work at the institute.

\section{REFERENCES}

[1] Richard Van Nee, Ramee Prasad, "OFDM for Wireless Multimedia Communication," Artech House universal personal communication library, Boston, London, pp. 33-37.

[2] Charan Langton, "Intuitive guide to principles of communication, Orthogonal Frequency Division Multiplexing Tutorial," Copyright 2004.

[3] J. Wang, X. Zhou, Y. Xu, W. Wang, "Performance Improvement of OFDM-ROF System with Clipping and Filtering Technique," IEEE Trans. on Consumer Electronics, vol. 54, no. 2, pp. 296-299, 2008

[4] S. A. Aburakhia, E. F. Badran, and D. A. E. Mohamed, "Linear Companding Transform for the Reduction of Peak-to-Average Power Ratio of OFDM Signals," IEEE Trans. on Broadcasting, vol. 55, no. 1, pp. 155-160, 2009.

[5] Y. Tsai, G. Zhang, and X. Wang, "Polyphase Codes for Uplink OFDM-CDMA Systems," IEEE Trans. on Communications, vol. 56, no. 3, pp. 435-444, 2008.

[6] R.F.H. Fischer, "Widely-linear selected mapping for peaktoaverage power ratio reduction in OFDM," Electronics Letters, vol. 43, no. 14, pp. 1-2, 2007.

[7] X. Wu, J. Wang, and Z. Mao, "A Novel PTS Architecture for PAPR Reduction of OFDM Signals," In proceedings of IEEE ICCS 2008, pp. 1055-1060, Guangzhou, China, November, 2008.

[8] A. Ghassemi, T. A. Gulliver, "A Low-Complexity PTSBased Radix FFT Method for PAPR Reduction in OFDM Systems," IEEE Trans. on Signal Processing, vol. 56, no. 3, pp. 1161-1166, 2008.

[9] T. T. Nguyen, and L. Lampe, "On Partial Transmit Sequences for PAR Reduction in OFDM Systems," IEEE Trans. on Wireless Communications, vol. 7, no. 2, pp. 746-755, 2008.

[10] Tao Jiang, and Yiyan Wu "An Overview: Peak-toAverage Power Ratio Reduction Techniques for OFDM Signals," IEEE Trans. on Broadcasting. vol.54, No.2, June 2008.

[11] ByungMooLee - Rui J. P. deFigueiredo - YoungokKim, A Computationally Efficient Tree-PTS Technique for PAPR Reduction of OFDM Signals", (C) Springer Science+Business Media, LLC. 2010.

[12] Xinchun Wu, Zhigang Mao, Jinxiang Wang, Bin Zhou, "A Novel PTS Technique with Combinative Optimization in Real Part and Imaginary Part for PAPR Reduction in OFDM Systems", 2009 Third International Conference on Next Generation Mobile Applications, Services and Technologies. 
[13] Tellambura, Chintha and Jayalath, Dhammika (2001) PAR reduction of an OFDM signal using partial transmit sequences. In Proceedings IEEE VTS 54th Vehicular Technology Conference, 2001 (VTC 2001 fall).

[14] Trung Thanh Nguyen and Lutz Lampe, "On Partial Transmit Sequences for PAR Reduction in OFDM Systems", Revised as Paper for publication in the IEEE Transactions on Wireless Communications, November, 2006.
[15] Chusit Pradabpet and Kobchai Dejhan, "A new PAPR reduction in OFDM systems using PTS combined with APPR for TWTA nonlinear HPA", Songklanakairn journal of Science and Technology. Received 4 April 2007; Accepted 1 May 2008.

[16] Jayalath, Dhammika and Tellambura, Chintha, "Adaptive PTS approach for reduction of peak-to-average power ratio of OFDM signa", ELECTRONICS LETTERS 6th July 2000 Vol. 36 No. 14, () Copyright 2000 IEEE. 